\title{
Effects of Mechanical Performance on Deliverability and Dose Distribution by Comparing Multi Institutions' Knowledge-based Models for Prostate Cancer in Volumetric Modulated Arc Therapy
}

\author{
HARUHI TSURU ${ }^{1,2}$, YOSHIHIRO UEDA ${ }^{1}$, MIKOTO TAMURA ${ }^{3}$, HAJIME MONZEN ${ }^{3}$, \\ SHINGO OHIRA ${ }^{1}$, AKIRA MASAOKA ${ }^{1}$, SHOUKI INUI ${ }^{1,2}$, KOJI KONISHI $^{1}$, JUNICHI FUKUNAGA ${ }^{4}$, \\ HIROKAZU MIZUNO ${ }^{5}$, MASAYOSHI MIYAZAKI ${ }^{1}$ and MASAHIKO KOIZUMI ${ }^{2}$ \\ ${ }^{1}$ Department of Radiation Oncology, Osaka International Cancer Institute, Osaka, Japan; \\ ${ }^{2}$ Department of Medical Physics and Engineering, Graduate School of Medicine, Osaka University, Osaka, Japan; \\ ${ }^{3}$ Department of Medical Physics, Graduate School of Medical Sciences, Kindai University, Osaka, Japan; \\ ${ }^{4}$ Division of Radiology, Department of Medical Technology, Kyushu University Hospital, Fukuoka, Japan; \\ ${ }^{5}$ Division of central radiology, Osaka Rosai Hospital, Osaka, Japan
}

\begin{abstract}
Background/Aim: The aim of this study was to evaluate the mechanical performance and the effect on dose distribution and deliverability of volumetric modulated arc therapy (VMAT) plans for prostate cancer created with the commercial knowledge-based planning (KBP) system (RapidPlan $\left.{ }^{T M}\right)$. Materials and Methods: Three institutions, $A, B$, and $C$ were enrolled in this study. Each institution established and trained a KBP model with their own cases. $C T$ data and structures for 45 patients at institution $B$ were utilized to validate the dose-volume parameters $\left(D_{2(\%)}\right.$,

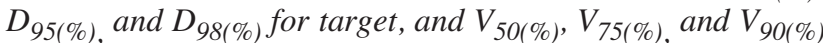
for rectum and bladder), and the following mechanical performance parameters and gamma passing rates of each KBP model: leaf sequence variability $(L S V)$, aperture area variability $(A A V)$, total monitor unit $(M U)$, modulation complexity score for VMAT (MCSv), MU/control point (CP), aperture area $(A A) / C P$, and $M U \times A A / C P$. Results: Significant differences $(p<0.01)$ in dosimetric parameters such as $D_{2}$ and $D_{98}$ for target and $V_{50}, V_{75}$, and $V_{90}$ for bladder were observed among the three institutions. The means and
\end{abstract}

This article is freely accessible online.

Correspondence to: Yoshinori Ueda, MS, Department of Radiation Oncology, Osaka International Cancer Institute, 3-1-69 Otemae, Chuo-ku, Osaka-shi, Osaka, 537-8567, Japan. Tel: +81 669451181, Fax: +81 669451900, e-mail: ueda-yo@mc.pref.osaka.jp

Key Words: Knowledge-based planning, multi models, MCSv, RapidPlan. standard deviations of MCSv were $0.31 \pm 0.03,0.29 \pm 0.02$, and $0.32 \pm 0.03$, and the angles of maximum and minimum $M U \times A A / C P$ were $269^{\circ}$ and $13^{\circ}, 269^{\circ}$ and $13^{\circ}$, and $273^{\circ}$ and $153^{\circ}$ at institutions $A, B$, and $C$, respectively. The mean gamma passing rate $(1 \% / 1 \mathrm{~mm}$.) was $>95 \%$ for all cases in each institution. Dose distribution and mechanical performance significantly differed between the three models. Conclusion: Each KBP model had different dose distributions and mechanical performance but could create an acceptable plan for deliverability regardless of mechanical performance.

Volumetric modulated arc therapy (VMAT) is an intensitymodulated technique delivered with dynamic gantry motion, while varying multi leaf collimators (MLC), dose rates, and gantry speeds (1) and can be used to create a steep dose gradient and complement dose distribution (2). It utilizes inverse planning to improve target conformity and organ at risk (OAR) sparing (3) and has often been used for prostate and head and neck cancer $(4,5)$. However, one of the disadvantages of VMAT is that the plan quality, such as target coverage and OAR sparing, depends on the planner's skill and experience or institution's plan policy during optimization $(6,7)$.

VMAT plans require more complex parameters related to treatment equipment such as gantry, linear accelerator, and MLC than conformal plans; therefore, it is recommended that patient-specific quality assurance (QA) be performed prior to initiating treatment to ensure deliverability. Complexity is associated with gantry speed, MU and sequence and aperture of MLC, which we defined as 
mechanical performance. One of the complex plans includes high MU, variable gantry speed and sequence and small aperture of MLC. Nowadays, various metrics to quantify mechanical performance have been developed, and it has been reported that some metrics relate to deliverability and indicate the possibility of passing QA for delivered plans $(1,8)$.

Knowledge-based planning (KBP) has an important role in standardizing plan quality. RapidPlan ${ }^{\mathrm{TM}}$ (Varian Medical Systems, Palo Alto, CA, USA) is a commercial KBP tool incorporated in the Eclipse treatment planning system. By learning the dosimetric and geometric information of the registered cases, RapidPlan ${ }^{\mathrm{TM}}$ predicts an achievable dosevolume histogram for the organ at risk and provides the optimal dose distribution for the new patients (9). If the institutions share better KBP models, the plan quality will improve and be standardized. Some studies have shown that KBP plans are acceptable for clinical use in various treatment sites (10-12). In one study, Tamura et al. evaluated the mechanical performance and dose accuracy of plans generated with a KBP model by comparing clinical plans, and found that the KBP system of VMAT for prostate cancer could create plans clinically acceptable for dose accuracy without any major problems (13).

When comparing the dosimetric parameters calculated by each KBP model for prostate cancer in multiple institutions, the performance in sparing OAR varied by the enrolled model $(6,14,15)$. However, Kubo et al. found that MU and MLC sequence complexity calculated with RapidPlan were higher than the clinical plan, and the possibility of passing QA may depend on the KBP model (9). There has been no study comparing mechanical performances calculated with multi-models in KBP. Moreover, we suggest quantifying the effect of MLC aperture and MU at every angle during one full arc on dose distribution as new metrics in evaluating whether to share the KBP model between institutions. This study aimed to evaluate mechanical performances and the effect on deliverability and dose distribution using the KBP models for prostate cancer of three institutions in VMAT.

\section{Materials and Methods}

Definitions of structures and planning design at each institution. Three institutions (Institutions A, B, and C) were enrolled in this study. Each KBP model was configured using clinical plans for patients with T1-T2c prostate cancer VMAT in each institution, which had different contouring definitions. The definitions of the clinical target volume (CTV) were the prostate and $15 \mathrm{~mm}$ of seminal vesicle (SV), the prostate and half of the SV, and the prostate and $10 \mathrm{~mm}$ of $\mathrm{SV}$ at institutions $\mathrm{A}, \mathrm{B}$, and $\mathrm{C}$, respectively. The planning target volume (PTV) was generated by adding an 8$\mathrm{mm}, 8-\mathrm{mm}$, and 6-mm margin around the CTV in all dimensions, except posteriorly, where 5-mm, 6- $\mathrm{mm}$, and 4-mm margins were used, at institutions A, B, and C, respectively. The definition for the rectum was up to $1.0 \mathrm{~cm}$ above and below the $\mathrm{PTV}, 1.5 \mathrm{~cm}$ above
Table I. Dose prescription and dose constraints for the VMAT planning in each institution.

\begin{tabular}{|c|c|c|c|c|}
\hline \multicolumn{5}{|c|}{ Dose constraints } \\
\hline Institution & Organ & & Target & $\begin{array}{c}\text { Prescribed } \\
\text { volume }\end{array}$ \\
\hline \multirow[t]{5}{*}{ A } & Rectal wall & Bladder wall & \multirow[t]{5}{*}{ CTV } & PTV-Rectum \\
\hline & V78Gy<1\% & V70Gy<35\% & & Dmean $<103 \%$ \\
\hline & V70Gy<20\% & V $40 \mathrm{~Gy}<60 \%$ & & Dmin $>99 \%$ \\
\hline & V60Gy<30\% & & & $\operatorname{Dmax}<110 \%$ \\
\hline & V $40 \mathrm{~Gy}<60 \%$ & & & $\mathrm{D} 95 \%=100 \%$ \\
\hline \multirow[t]{5}{*}{ B } & Rectal wall & Bladder wall & \multirow[t]{5}{*}{ CTV } & PTV \\
\hline & $\mathrm{V} 78 \mathrm{~Gy} \leq 1 \%$ & V70Gy $\leq 35 \%$ & & Dmean $=100 \%$ \\
\hline & V70Gy $\leq 20 \%$ & V $40 \mathrm{~Gy} \leq 60 \%$ & & D95\% $\geq 95 \%$ \\
\hline & V60Gy $\leq 35 \%$ & & & V $90 \% \geq 98 \%$ \\
\hline & V $40 \mathrm{~Gy} \leq 60 \%$ & & & Dmax $\leq 110 \%$ \\
\hline \multirow[t]{5}{*}{$\mathrm{C}$} & Rectal wall & Bladder wall & CTV & PTV \\
\hline & V70Gy $\leq 5 \%$ & $\mathrm{~V} 80 \mathrm{~Gy} \leq 5 \%$ & D $98 \% \geq 98 \%$ & Dmean $=100 \%$ \\
\hline & V $65 \mathrm{~Gy} \leq 10 \%$ & V75Gy $\leq 15 \%$ & \multirow[t]{3}{*}{$\mathrm{D} 2 \% \leq 105 \%$} & D95\% $\geq 95 \%$ \\
\hline & V60Gy $\leq 20 \%$ & V70Gy $\leq 25 \%$ & & $\mathrm{~V} 90 \% \geq 98 \%$ \\
\hline & V $40 \mathrm{~Gy} \leq 40 \%$ & V $60 \mathrm{~Gy} \leq 40 \%$ & & $\mathrm{D} 2 \% \leq 105 \%$ \\
\hline
\end{tabular}

CTV: Clinical target volume; PTV: planning target volume. Rectal/Bladder wall: the volume with a wall thickness of $4 \mathrm{~mm}$ generated automatically from the rectum/bladder contour

the seminal vesicles to $1.5 \mathrm{~cm}$ below the prostate, and the tissue extending from the rectosigmoid junction to the anus at institutions $\mathrm{A}, \mathrm{B}$, and $\mathrm{C}$, respectively. Table I shows dose prescription and dose constraints for the VMAT planning in each institution.

In each institution, the model for KBP was created using that institution's VMAT plans for clinical use before April 2017. At institution A, the PTV minus the rectum was used as the prescribed volume (PV). In institutions B and C, the PTV was used as the PV. The prescription dose was a mean PTV dose of 78 Gy at institutions $\mathrm{B}$ and $\mathrm{C}$, and a minimum dose to $95 \%\left(\mathrm{D}_{95}\right)$ of the PTV minus the rectum at institution $\mathrm{A}$. The number of registered cases in institution A, B, and C was 50, 100, and 20, respectively. Each model was clinically accepted at each institution and sent to institution B.

Validation plans for KBP. To validate the performance of KBP at each institution, 45 prostate cancer cases who were clinically treated from May 2017 to April 2018 at institution B were used in a single optimization with the RapidPlan ${ }^{\mathrm{TM}}$ system. For these validation plans (VP), a beam energy of $6 \mathrm{MV}$ photons from a TrueBeam STx linear accelerator equipped with a high definition 120-leaf multileaf collimator (MLC) (Varian Medical Systems) was utilized. The treatment field was one full arc rotating counterclockwise from $179^{\circ}$ to $181^{\circ}$, with a collimator rotation of $30^{\circ}$. Institution B accepts one full arc for prostate cancer because dose distribution and time efficiency in one full arc are comparable to and better than that in two - three arc. After calculation, MLC leaf position, gantry speed, and dose rate were defined by each control point (CP) and CPs were spaced $2^{\circ}$ apart in one full arc. The optimization and calculation algorithms used were the Anisotropic Analytical Algorithm and Photon Optimizer 13.0 (Varian Medical Systems) with Eclipse ver. 13.5 , and the grid size was $2.5 \mathrm{~mm}$. The prescription setting for the 
VP of each model was the same as that of the clinical plan in each institution. Written informed consent was obtained from all patients, and the Institutional Ethics Committee approved this study (Osaka International Cancer Institute review board number: 1611119172).

Dosimetric data analysis. The dose-volume relationship, represented by the dose as a percentage of the prescribed dose to $2.0 \%, 95 \%$, and $98 \%$ of the $\mathrm{PV}\left(\mathrm{D}_{2}, \mathrm{D}_{95}\right.$, and $\mathrm{D}_{98}$, respectively) and the volume ratio as receiving $50 \%, 75 \%$, and $90 \%$ of the prescribed dose $\left(\mathrm{V}_{50}, \mathrm{~V}_{75}\right.$, and $\mathrm{V}_{90}$, respectively) for the rectum and bladder, was extracted from dose-volume histogram data for VP with each model.

Mechanical performance. To analyze the mechanical performance of the VP, leaf sequence variability (LSV), aperture area variability (AAV), modulation complexity score for VMAT (MCSv), and total monitor units (MU) during one full arc were calculated using inhouse software created by MATLAB R2016a (MathWorks, Natick, MA, USA). MCSv is a normalized sum over all CPs of LSV and AAV. The MCSv, LSV, and AAV values range from 0 to 1 and a small value indicates that the MLC motion is complex (1). When the value of LSV and AAV is 1, the field shape is rectangular, and the aperture area equals the maximum aperture area in one full arc. On the other hand, when the value of LSV and AAV is approximately 0 , the differences in position between adjacent MLC leaves are large and the aperture area is much smaller than the maximum aperture area in one full arc. In this study, we adopted the mean of these values during one full arc.

In each plan, changes in $\mathrm{MU}$, area aperture (AA) and the product of $\mathrm{MU}$ and $\mathrm{AA}$ (MU×AA) at each $\mathrm{CP}$ were evaluated and represented as MU/CP, AA/CP and MU×AA/CP. Using 45 cases in each institution, the means at every $\mathrm{CP}$, along with the mean and standard deviation (SD) considering all CPs were calculated.

Gamma analysis. We measured the dose response of the three institutions' plans with an electronic portal imaging device (EPID) detector, aS1200, equipped with a TrueBeam STx linear accelerator. The total area and matrix size of the EPID were $40 \times 40 \mathrm{~cm}^{2}$ and $1,190 \times 1,190$ pixels, respectively. All EPID images were acquired in the integrated acquisition mode with a source-to-imager distance of $160 \mathrm{~cm}$. Measured dose responses were compared with planned dose responses using global gamma analysis. The gamma analysis was performed with a criterion of $1 \% / 1 \mathrm{~mm}$ (dose difference and distance to agreement) and a threshold at $10 \%$ using the commercial software PerFraction (SUN Nuclear corporation, Melbourne, FL, USA) because all plans in the three institutions are $100 \%$ in conditions $3 \% / 3 \mathrm{~mm}$ and $2 \% / 2 \mathrm{~mm}$.

Statistical analysis. The paired Wilcoxon signed rank test was used to compare dosimetric parameters, mechanical performance, and passing rate of gamma analysis between two of the three institutions. A $p$-value of $<0.05$ was considered significant.

\section{Results}

Table II summarizes the results comparing $\mathrm{D}_{2}, \mathrm{D}_{95}$, and $\mathrm{D}_{98}$ for the $\mathrm{PV}$ and $\mathrm{V}_{50}, \mathrm{~V}_{75}$, and $\mathrm{V}_{90}$ for the rectum and bladder across the three institutions. For PV, there was a significant difference $(p<0.01)$ between each paired institution, except for $\mathrm{D}_{95}$ between institutions B and C. Institution A had the highest PV among the three institutions, whereas institution B had the lowest.
Table II. The summary of dose-volume parameters in three institutions.

\begin{tabular}{lccc}
\hline & Institution A & Institution B & Institution C \\
\cline { 2 - 4 } PV & PTV-Rectum & PTV & PTV \\
\hline $\mathrm{D}_{2}$ & $104.5 \% \pm 0.9 \%$ & $102.4 \% \pm 0.4 \%$ & $102.8 \% \pm 0.4 \%$ \\
$\mathrm{D}_{95}$ & $100.0 \% \pm 0.4 \%$ & $96.4 \% \pm 0.5 \%$ & $96.5 \% \pm 0.5 \%$ \\
$\mathrm{D}_{98}$ & $99.0 \% \pm 0.3 \%$ & $94.1 \% \pm 0.8 \%$ & $94.4 \% \pm 0.7 \%$ \\
\hline
\end{tabular}

\section{Rectum}

\begin{tabular}{lccc}
\hline $\mathrm{V}_{50}$ & $40.2 \% \pm 7.7 \%$ & $40.3 \% \pm 6.8 \%$ & $31.5 \% \pm 10.5 \%$ \\
$\mathrm{~V}_{75}$ & $19.1 \% \pm 5.0 \%$ & $23.7 \% \pm 7.5 \%$ & $19.6 \% \pm 8.9 \%$ \\
$\mathrm{~V}_{90}$ & $9.6 \% \pm 3.0 \%$ & $13.8 \% \pm 6.1 \%$ & $11.7 \% \pm 6.2 \%$ \\
\hline
\end{tabular}

\section{Bladder}

\begin{tabular}{lccc}
\hline $\mathrm{V}_{50}$ & $37.7 \% \pm 14.4 \%$ & $29.1 \% \pm 14.2 \%$ & $33.6 \% \pm 15.0 \%$ \\
$\mathrm{~V}_{75}$ & $20.1 \% \pm 9.7 \%$ & $16.3 \% \pm 9.3 \%$ & $18.1 \% \pm 8.6 \%$ \\
$\mathrm{~V}_{90}$ & $13.8 \% \pm 7.3 \%$ & $11.3 \% \pm 7.1 \%$ & $12.1 \% \pm 6.2 \%$ \\
\hline
\end{tabular}

\begin{tabular}{lccr}
\hline & \multicolumn{3}{c}{ Institution comparison $p$-Values } \\
\cline { 2 - 4 } & $\mathrm{A} \times \mathrm{B}$ & $\mathrm{A} \times \mathrm{C}$ & $\mathrm{B} \times \mathrm{C}$ \\
\hline $\mathrm{PV}$ & $\mathrm{PTV}$-Rectum & $\mathrm{PTV}$ & $\mathrm{PTV}$ \\
\hline $\mathrm{D}_{2}$ & $<0.01$ & $<0.01$ & $<0.01$ \\
$\mathrm{D}_{95}$ & $<0.01$ & $<0.01$ & 0.38 \\
$\mathrm{D}_{98}$ & $<0.01$ & $<0.01$ & $<0.01$ \\
\hline
\end{tabular}

Rectum

\begin{tabular}{lrrr}
\hline $\mathrm{V}_{50}$ & 0.37 & $<0.01$ & $<0.01$ \\
$\mathrm{~V}_{75}$ & $<0.01$ & 0.45 & $<0.01$ \\
$\mathrm{~V}_{90}$ & $<0.01$ & $<0.05$ & $<0.01$ \\
\hline
\end{tabular}

\section{Bladder}

\begin{tabular}{llll}
\hline $\mathrm{V}_{50}$ & $<0.01$ & $<0.01$ & $<0.01$ \\
$\mathrm{~V}_{75}$ & $<0.01$ & $<0.01$ & $<0.01$ \\
$\mathrm{~V}_{90}$ & $<0.01$ & $<0.01$ & $<0.01$ \\
\hline
\end{tabular}

PTV: Planning target volume; PV: prescribed volume.

In the dosimetric parameters for the rectum, there was a significant difference $(p<0.01$ or 0.05$)$ between each paired institution, except for $\mathrm{V}_{50}$ between institutions $\mathrm{A}$ and $\mathrm{B}$, and $V_{75}$ between institutions $A$ and $C . V_{75}$ and $V_{90}$ for institution $A$, and $\mathrm{V}_{50}$ for institution $\mathrm{C}$ were the lowest among the three institutions for the rectum. Regarding the dosimetric parameters for the bladder, there were significant differences $(p<0.01)$ between all pairs. Institution $\mathrm{B}$ had the lowest bladder results, whereas institution $\mathrm{C}$ had the highest.

Figure 1 shows the dose distribution in each institution. Institution A had the highest uniformity for a $100 \%$ PTV isodose. The lower line of a $50 \%$ isodose for institution $\mathrm{C}$ 

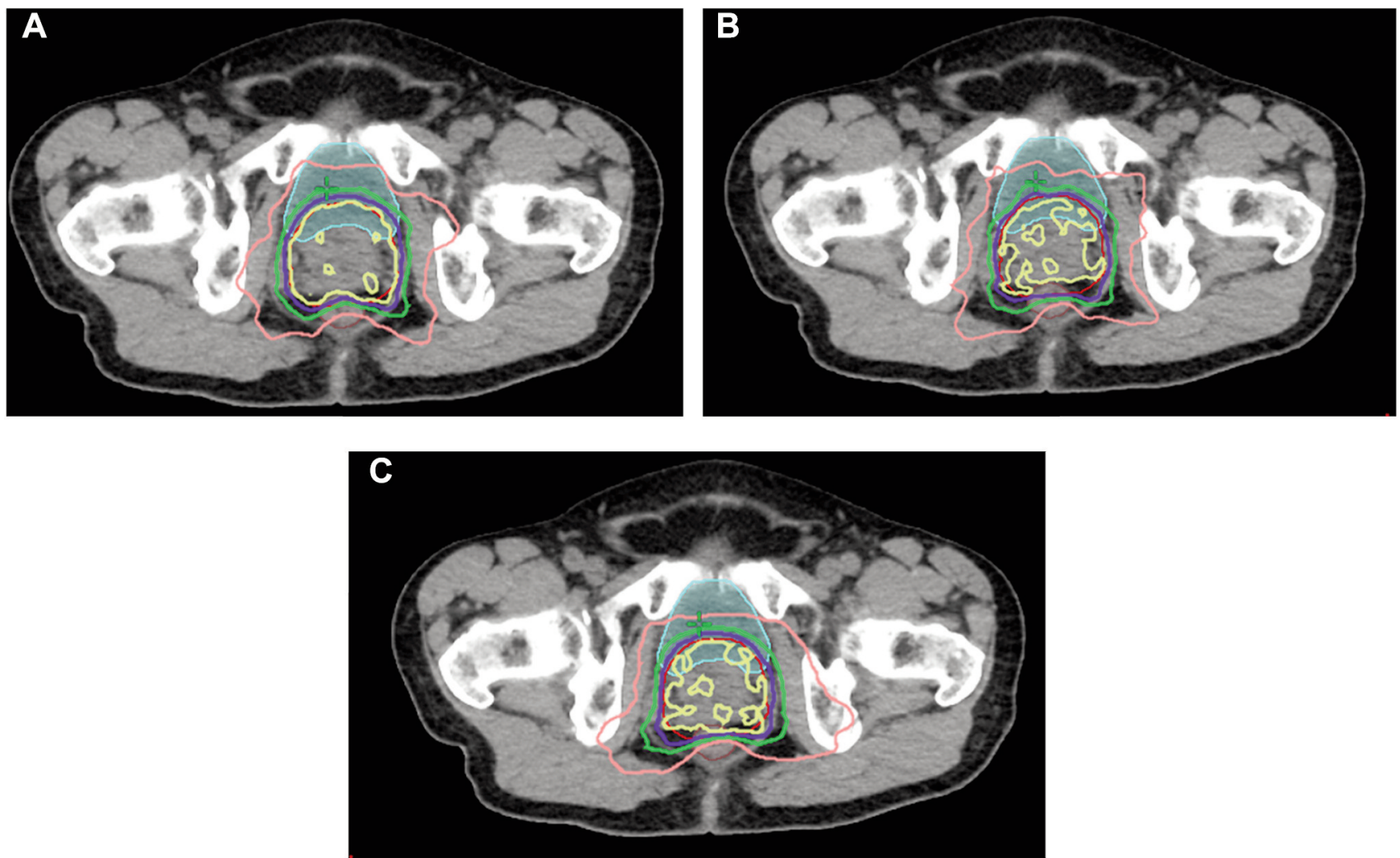

Figure 1. Comparison of the dose distribution from three institutions' plans. (A), (B) and (C) show the dose distributions of institutions A, B, and C. Yellow, blue, green, and pink curves indicate 100\%, 90\%, 75\%, and 50\% isodose curves, respectively. Red, brown, and light blue curves indicate planning target volume, rectum, and bladder, respectively.

expanded the most widely horizontally, especially on the left side. Considering this fact, each model created a characteristic dose distribution.

Table III shows the mean, SD and range $(\mathrm{max} / \mathrm{min})$ of the $\mathrm{LSV}, \mathrm{AAV}$, total MU, and MCSv, as well as the $p$-value of each mechanical performance in the three groups. Institution $\mathrm{C}$ had the highest LSV and there were significant differences in the group that comprises institution $\mathrm{C}$ (A vs. C and B vs. C). Institution B had the lowest AAV and MCSv and the highest total MU. There were significant differences $(p<0.01$ or 0.05 ) in the group that comprises institution B (A vs. B and $\mathrm{B} v s . \mathrm{C}$ ) for $\mathrm{AAV}$ and total MU, and in all groups for MCSv. We found institution B used the closest area of MLC and the most complex modulation.

Figure 2 shows the MU/CP, AA/CP, and MU×AA/CP of the three institutions to investigate variation. Table IV shows the mean, $\mathrm{SD}$, and range ( $\mathrm{max} / \mathrm{min}$ ) of the $\mathrm{MU} / \mathrm{CP}, \mathrm{AA} / \mathrm{CP}$, and MU×AA/CP among the three institutions from $179^{\circ}$ to $181^{\circ}$ and the $p$ values of the three groups. The mean value for the MU/CP in each institution was the same. Institution A had the highest SD and the narrowest range for MU/CP in any parameter. Institution $\mathrm{B}$ had the lowest $\mathrm{AA} / \mathrm{CP}$ and
MU×AA/CP. The SD and maximum value for $\mathrm{MU} \times \mathrm{AA} / \mathrm{CP}$ in institution $\mathrm{C}$ were the highest of the three institutions. Significant differences $(p<0.01$ or 0.05$)$ were observed in all parameters across institutions.

The angle of maximum MU/CP was $101^{\circ}, 99^{\circ}$, and $101^{\circ}$ for institutions $\mathrm{A}, \mathrm{B}$, and $\mathrm{C}$, respectively. The angle of minimum $\mathrm{AA} / \mathrm{CP}$ was $13^{\circ}$ for all institutions. The angles of maximum and minimum MU×AA/CP were $269^{\circ}$ and $13^{\circ}$ for institution $\mathrm{A}, 269^{\circ}$ and $13^{\circ}$ for institution $\mathrm{B}$, and $273^{\circ}$ and $153^{\circ}$ for institution $\mathrm{C}$. In addition to minimum MU×AA/CP, $179^{\circ}$ and $181^{\circ}$ were not considered because MU/CP was much lower there. The maximum differences of $\mathrm{MU} / \mathrm{CP}$, $\mathrm{AA} / \mathrm{CP}$, and $\mathrm{MU} \times \mathrm{AA} / \mathrm{CP}$ at each angle were calculated among three institutions. The angle at which the maximum difference was the highest in 1 full arc was $99^{\circ}(0.27 \%), 1^{\circ}$ $\left(7.0 \mathrm{~cm}^{2}\right)$ and $275^{\circ}\left(5.7 \% \mathrm{~cm}^{2}\right)$, respectively.

For institution $\mathrm{A}, \mathrm{B}$, and $\mathrm{C}$, the gamma passing rate was $99.4 \% \pm 0.6 \%, 98.9 \% \pm 1.0 \%$ and $99.5 \% \pm 0.7 \%($ mean \pm SD), respectively, and was $>95 \%$ for all cases with a criterion of $1 \% / 1 \mathrm{~mm}$. There were significant differences $(p<0.01)$ in gamma passing rate between the groups pairing institution $\mathrm{B}$ (A vs. B and B vs. C). 

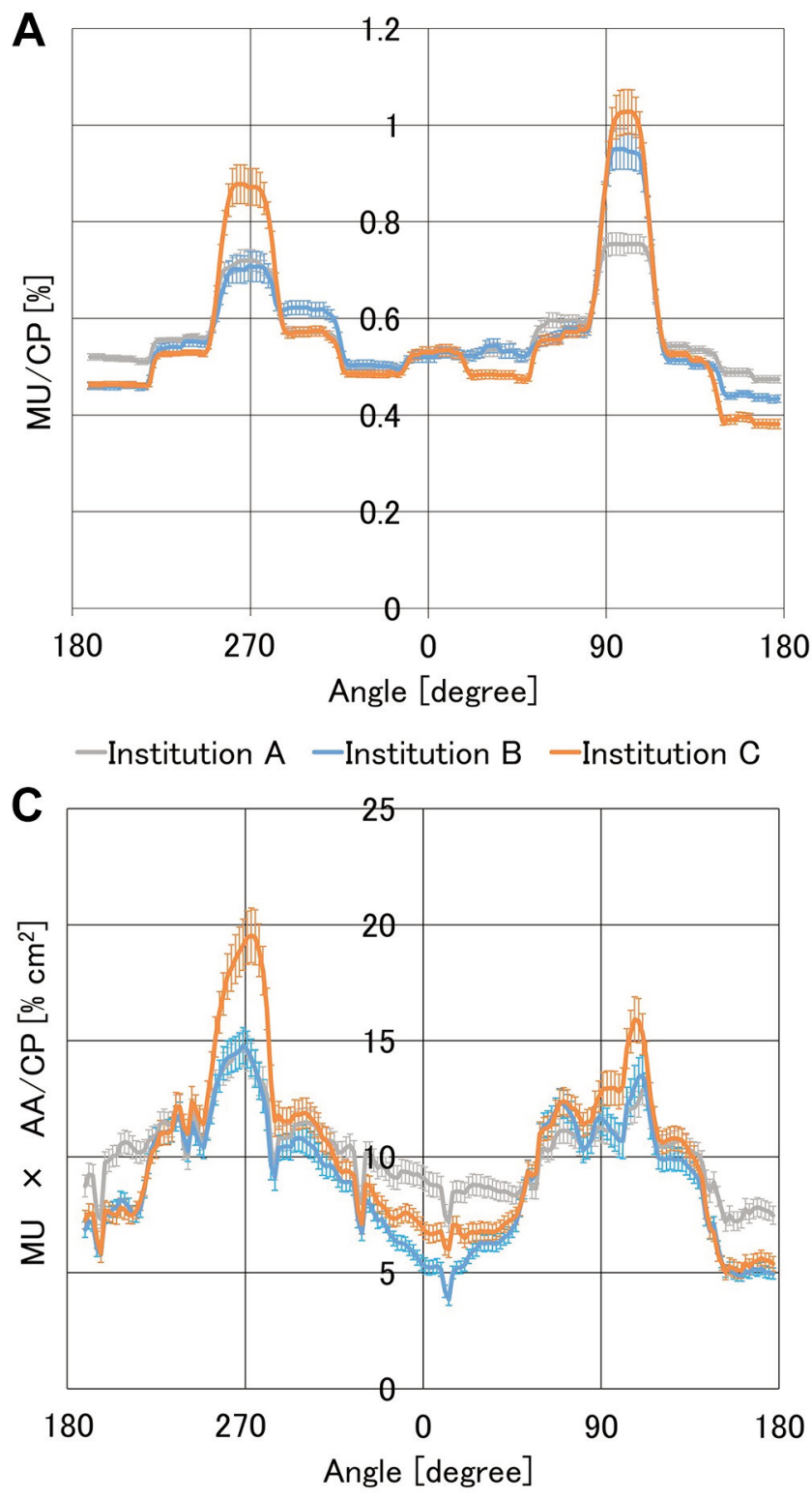

- Institutiton A - Institutiton B - Institutiton C

\section{Discussion}

In this study, we evaluated mechanical performance using prostate cancer models in VMAT and compared the complexity of MLC motion and dose distribution across three institutions. Regarding complexity, significant differences in MCSv were observed, but the plans were deemed clinically acceptable despite MCSv affecting gamma passing rate (1). The dose distribution of each institution met institution B's dose constraint and significant differences in dosimetric parameters of the target and OAR were observed. By quantifying MLC motion in each CP, through MU/CP,

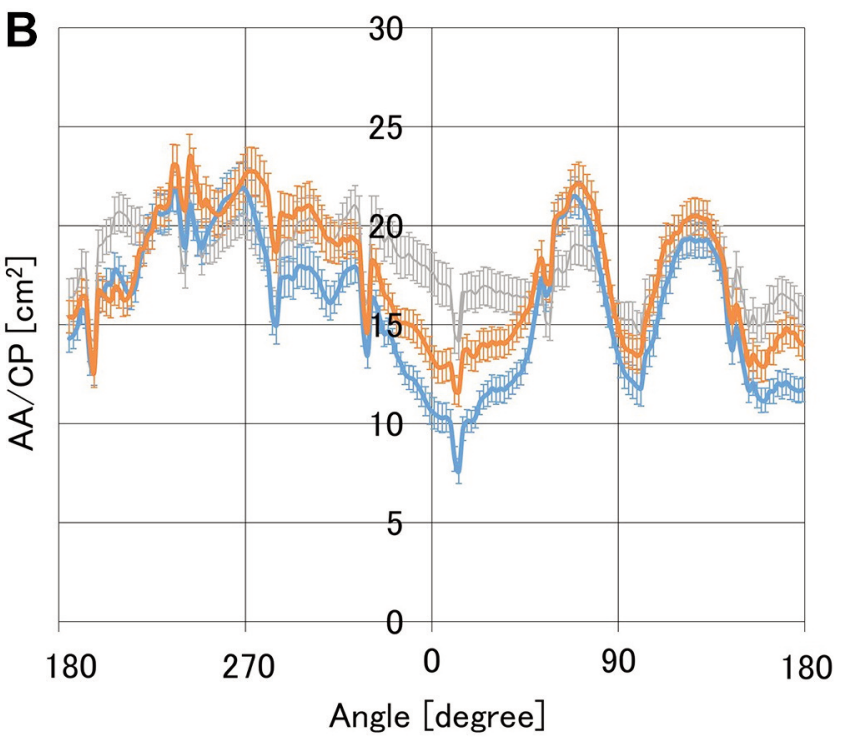

- Institution A - Institution B - Institution C

Figure 2. Comparison of $(A) M U / C P,(B)$ area aperture $(A A) / C P,(C)$ $M U \times A A / C P$ as a function of gantry angle in three institutions. The vertical axis represents the $M U$ and the horizontal axis represents the gantry angle. $M U, A A$, or MUXAA of each $C P$ averaged across 45 plans was plotted for each institution. Error bars represent the standard error. MU: Monitor unit; CP: control point; AA: aperture area.

$\mathrm{AA} / \mathrm{CP}$ and $\mathrm{MU} \times \mathrm{AA} / \mathrm{CP}$, it was found that the tendencies of the beam irradiation in each gantry angle varied between models and might link to OAR sparing. In summary, KBP model plans were clinically acceptable for deliverability regardless of the structure set. However, there is a need to select models considering individual institutional plan design because it affects dose distribution to the target and OAR by MLC motion.

Institution B obtained the lowest result for MCSv and gamma passing rate and the highest result for total MU among the three institutions, thus its model created the most complex plans in which the dosimetric accuracy is the 
Table III. Comparison of mechanical performance during all CPs in three institutions' models.

\begin{tabular}{|c|c|c|c|c|}
\hline Institution & $\begin{array}{c}\text { LSV } \\
(\max / \min )\end{array}$ & $\begin{array}{c}\text { AAV } \\
(\max / \min )\end{array}$ & $\begin{array}{c}\text { MCSv } \\
(\mathrm{max} / \mathrm{min})\end{array}$ & $\begin{array}{l}\text { Total MU } \\
\text { (max/min) }\end{array}$ \\
\hline A & $\begin{array}{c}0.72 \pm 0.06 \\
(0.89 / 0.59)\end{array}$ & $\begin{array}{c}0.44 \pm 0.04 \\
(0.50 / 0.35)\end{array}$ & $\begin{array}{c}0.31 \pm 0.03 \\
(0.38 / 0.23)\end{array}$ & $\begin{array}{c}681 \pm 66 \\
(947 / 573)\end{array}$ \\
\hline B & $\begin{array}{c}0.72 \pm 0.06 \\
(0.89 / 0.62)\end{array}$ & $\begin{array}{c}0.40 \pm 0.03 \\
(0.46 / 0.34)\end{array}$ & $\begin{array}{c}0.29 \pm 0.02 \\
(0.35 / 0.23)\end{array}$ & $\begin{array}{c}763 \pm 48 \\
(865 / 641)\end{array}$ \\
\hline $\mathrm{C}$ & $\begin{array}{c}0.73 \pm 0.05 \\
(0.89 / 0.62)\end{array}$ & $\begin{array}{c}0.44 \pm 0.02 \\
(0.51 / 0.34)\end{array}$ & $\begin{array}{c}0.32 \pm 0.03 \\
(0.39 / 0.24)\end{array}$ & $\begin{array}{c}678 \pm 44 \\
(793 / 583)\end{array}$ \\
\hline $\begin{array}{l}\text { Institution } \\
\text { Comparison }\end{array}$ & $\begin{array}{c}\text { LSV } \\
p \text {-Value }\end{array}$ & $\begin{array}{c}\text { AAV } \\
p \text {-Value }\end{array}$ & $\begin{array}{c}\text { MCSv } \\
p \text {-Value }\end{array}$ & $\begin{array}{c}\text { Total MU } \\
p \text {-Value }\end{array}$ \\
\hline A $v s . \mathrm{B}$ & 0.28 & $<0.01$ & $<0.01$ & $<0.01$ \\
\hline A $v s . C$ & $<0.01$ & 0.41 & $<0.05$ & 0.35 \\
\hline B vs. C & $<0.01$ & $<0.01$ & $<0.01$ & $<0.01$ \\
\hline
\end{tabular}

LSV: Leaf sequence variability; AAV aperture area variability; MCSv: modulation complexity score for VMAT; MU: monitor unit.

lowest. In one study by Sarah et al., when the modulation complexity of score (MCS) for a step-and-shoot IMRT static beam was greater than 0.35 (MCS $>0.35$ ), all gamma passing rates $(3 \% / 3 \mathrm{~mm})$ were greater than $95 \%$ (16). In this study, the gamma passing rates were $>95 \%$ for all cases in each institution for the criterion of $1 \% / 1 \mathrm{~mm}$, while some plans had MCSv $<0.35$, thus KBP plans were applied clinically without any major problem regardless of mechanical performance. It was assumed that the models could create the plans with high dosimetric accuracy (gamma passing rate) because clinically acceptable cases registered in each model in this study had simple MLC aperture. If models consisting of complex cases create the plans, the gamma passing rate may decrease.

Among VP with each model, we compared MU/CP, AA/CP, and $\mathrm{MU} \times \mathrm{AA} / \mathrm{CP}$ at each gantry angle. In Figure 2, the standard error of three parameters for each model were small, and the beam irradiation hardly changed in 45 plans. The angle for institutions $\mathrm{A}$ and $\mathrm{B}$ where MU×AA/CP reached its maximum and minimum was about $270^{\circ}$ and $10^{\circ}$, respectively, while the angle for institution $\mathrm{C}$ was about $270^{\circ}$ and $150^{\circ}$. Each model created plans where the irradiation intensity from lateral direction was higher because of avoiding the rectum. However, focusing on MU×AA/CP for institution A and C, there is a difference in the tendency of the beam irradiation. Institution A irradiated the most evenly at any angle as can be seen from Figure 2 because SD and range for institution A were lower and narrower. On the other hand, institution $\mathrm{C}$ also had higher MU×AA/CP at approximately $90^{\circ}$ and $270^{\circ}$, and lower $\mathrm{MU} \times \mathrm{AA} / \mathrm{CP}$ at approximately $0^{\circ}$ and $180^{\circ}$ than institution A. Therefore, the difference in the tendency of the
Table IV. Comparison of mechanical performance ( $M U, A A, M U \times A A)$ at each CP from $179^{\circ}$ to $181^{\circ}$ between three institutions.

\begin{tabular}{lccc}
\hline Institution & $\begin{array}{c}\mathrm{MU} / \mathrm{CP}(\%) \\
\text { range }(\mathrm{max} / \mathrm{min})\end{array}$ & $\begin{array}{c}\mathrm{AA} / \mathrm{CP}\left(\mathrm{cm}^{2}\right) \\
\text { range }(\mathrm{max} / \mathrm{min})\end{array}$ & $\begin{array}{c}\mathrm{MU} \times \mathrm{AA} / \mathrm{CP}\left(\% \mathrm{~cm}^{2}\right) \\
\text { range }(\mathrm{max} / \mathrm{min})\end{array}$ \\
\hline $\mathrm{A}$ & $0.56 \pm 0.08$ & $18.1 \pm 1.8$ & $10.1 \pm 1.8$ \\
& $(0.75 / 0.23)$ & $(21.7 / 14.2)$ & $(14.7 / 3.7)$ \\
$\mathrm{B}$ & $0.56 \pm 0.12$ & $15.9 \pm 3.6$ & $8.9 \pm 2.8$ \\
& $(0.95 / 0.22)$ & $(21.9 / 7.6)$ & $(14.8 / 2.5)$ \\
$\mathrm{C}$ & $0.56 \pm 0.16$ & $17.6 \pm 3.1$ & $10.0 \pm 3.5$ \\
& $(1.03 / 0.19)$ & $(23.4 / 11.6)$ & $(19.5 / 2.7)$ \\
\hline Institution & $\mathrm{MU} / \mathrm{CP}$ & $\mathrm{AA} / \mathrm{CP}$ & $\mathrm{MU} \times \mathrm{AA} / \mathrm{CP}$ \\
Comparison & $p$-Value & $p$-Value & $p$-Value \\
\hline A vs. B & $<0.01$ & $<0.01$ & $<0.01$ \\
A v s. C & $<0.01$ & $<0.01$ & $<0.05$ \\
B vs. C & $<0.01$ & $<0.01$ & $<0.01$ \\
\hline
\end{tabular}

MU: Monitor unit; AA: aperture area.

beam irradiation affected model's sparing performance where the mean of $V_{50}$ for rectum in institution $\mathrm{C}$ was the lowest of the three institutions, and the 50\% isodose curve of institution C extended more widely than that of institution A. Evaluating some plans created with a model in order to understand the model feature will serve as a tool for model sharing between institution.

\section{Conclusion}

Each KBP model had different dose distributions and mechanical performance but could create an acceptable plan for deliverability regardless of mechanical performance. MLC aperture and MU at every CP indicated institution's tendency of beam irradiation to understand model feature.

\section{Conflicts of Interest}

No conflicts of interest exist regarding this study.

\section{Authors' Contributions}

Software development: Tsuru H, Masaoka A, Ueda Y, Ohira S; Research design: Tsuru H, Ueda Y, Tamura M, Monzen H, Mizuno H, Miyazaki M; Manuscript writing: Tsuru H, Ueda Y, Konishi K, Inui S, Koizumi M.

\section{Acknowledgements}

This work was supported by a Grant-in Aid for Scientific Research (C) (grant number JP18K07632) and a JSPS KAKENHI Grant (grant number $21 \mathrm{~K} 07728$ ). This study was funded by a Japanese Society of Radiological Technology (JSRT) Research Grant $(2019,2020)$. 


\section{References}

1 Masi L, Doro R, Favuzza V, Cipressi S and Livi L: Impact of plan parameters on the dosimetric accuracy of volumetric modulated arc therapy. Med Phys 40(7): 071718, 2013. PMID: 23822422. DOI: $10.1118 / 1.4810969$

2 Otto K: Volumetric modulated arc therapy: IMRT in a single gantry arc. Med Phys 35(1): 310-317, 2008. PMID: 18293586. DOI: $10.1118 / 1.2818738$

3 Intensity Modulated Radiation Therapy Collaborative Working Group: Intensity-modulated radiotherapy: current status and issues of interest. Int J Radiat Oncol Biol Phys 51(4): 880-914, 2001. PMID: 11704310. DOI: 10.1016/s0360-3016(01)01749-7

4 Sale C and Moloney P: Dose comparisons for conformal, IMRT and VMAT prostate plans. J Med Imaging Radiat Oncol 55(6): 611-621, 2011. PMID: 22141609. DOI: 10.1111/j.1754-9485.2011.02310.x

5 Studenski MT, Bar-Ad V, Siglin J, Cognetti D, Curry J, Tuluc M and Harrison AS: Clinical experience transitioning from IMRT to VMAT for head and neck cancer. Med Dosim 38(2): 171-175, 2013. PMID: 23246253. DOI: 10.1016/j.meddos.2012.10.009

6 Ueda Y, Fukunaga JI, Kamima T, Adachi Y, Nakamatsu K and Monzen H: Evaluation of multiple institutions' models for knowledge-based planning of volumetric modulated arc therapy (VMAT) for prostate cancer. Radiat Oncol 13(1): 46, 2018. PMID: 29558940. DOI: 10.1186/s13014-018-0994-1

7 Wuthrick EJ, Zhang Q, Machtay M, Rosenthal DI, Nguyen-Tan PF, Fortin A, Silverman CL, Raben A, Kim HE, Horwitz EM, Read NE, Harris J, Wu Q, Le QT and Gillison ML: Institutional clinical trial accrual volume and survival of patients with head and neck cancer. J Clin Oncol 33(2): 156-164, 2015. PMID: 25488965. DOI: $10.1200 / J C O .2014 .56 .5218$

8 Tamura M, Monzen H, Matsumoto K, Kubo K, Ueda Y, Kamima $\mathrm{T}$, Inada M, Doi H, Nakamatsu K and Nishimura Y: Influence of cleaned-up commercial knowledge-based treatment planning on volumetric-modulated arc therapy of prostate cancer. J Med Phys 45(2): 71-77, 2020. PMID: 32831489. DOI: 10.4103/jmp. JMP_109_19

9 Kubo K, Monzen H, Ishii K, Tamura M, Kawamorita R, Sumida I, Mizuno $\mathrm{H}$ and Nishimura Y: Dosimetric comparison of RapidPlan and manually optimized plans in volumetric modulated arc therapy for prostate cancer. Phys Med 44: 199204, 2017. PMID: 28705507. DOI: 10.1016/j.ejmp.2017.06.026

10 Ueda Y, Miyazaki M, Sumida I, Ohira S, Tamura M, Monzen H, Tsuru $\mathrm{H}$, Inui $\mathrm{S}$, Isono $\mathrm{M}$, Ogawa $\mathrm{K}$ and Teshima $\mathrm{T}$ : Knowledgebased planning for oesophageal cancers using a model trained with plans from a different treatment planning system. Acta Oncol 59(3): 274-283, 2020. PMID: 31755332. DOI: 10.1080/ 0284186X.2019.1691257
11 Kamima T, Ueda Y, Fukunaga JI, Shimizu Y, Tamura M, Ishikawa $\mathrm{K}$ and Monzen $\mathrm{H}$ : Multi-institutional evaluation of knowledge-based planning performance of volumetric modulated arc therapy (VMAT) for head and neck cancer. Phys Med 64: 174-181, 2019. PMID: 31515017. DOI: 10.1016/j.ejmp. 2019.07.004

12 Kubo K, Monzen H, Ishii K, Tamura M, Nakasaka Y, Kusawake M, Kishimoto S, Nakahara R, Matsuda S, Nakajima T and Kawamorita R: Inter-planner variation in treatment-plan quality of plans created with a knowledge-based treatment planning system. Phys Med 67: 132-140, 2019. PMID: 31706149. DOI: 10.1016/j.ejmp.2019.10.032

13 Tamura M, Monzen H, Matsumoto K, Kubo K, Otsuka M, Inada M, Doi H, Ishikawa K, Nakamatsu K, Sumida I, Mizuno H, Yoon DK and Nishimura Y: Mechanical performance of a commercial knowledge-based VMAT planning for prostate cancer. Radiat Oncol 13(1): 163, 2018. PMID: 30170614. DOI: 10.1186/s13014-018-1114-y

14 Ueda Y, Monzen H, Fukunaga JI, Ohira S, Tamura M, Suzuki O, Inui S, Isono M, Miyazaki M, Sumida I, Ogawa K and Teshima T: Characterization of knowledge-based volumetric modulated arc therapy plans created by three different institutions' models for prostate cancer. Rep Pract Oncol Radiother 25(6): 10231028, 2020. PMID: 33390859. DOI: 10.1016/j.rpor.2020.08.011

15 Monzen H, Tamura M, Ueda Y, Fukunaga JI, Kamima T, Muraki Y, Kubo K and Nakamatsu K: Dosimetric evaluation with knowledge-based planning created at different periods in volumetric-modulated arc therapy for prostate cancer: a multiinstitution study. Radiol Phys Technol 13(4): 327-335, 2020. PMID: 32986184. DOI: 10.1007/s 12194-020-00585-0

16 Ghandour S, Matzinger O and Pachoud M: Volumetricmodulated arc therapy planning using multicriteria optimization for localized prostate cancer. J Appl Clin Med Phys 16(3): 5410, 2015. PMID: 26103500. DOI: 10.1120/jacmp.v16i3.5410
Received January 12, 2022

Revised January 29, 2022

Accepted January 31, 2022 